\title{
Spectral analysis on pseudo-Riemannian locally symmetric spaces
}

\author{
By Fanny KASSEL ${ }^{*)}$ and Toshiyuki KOBAYASHI ${ }^{* * * * * *)}$ \\ (Communicated by Masaki KashiwarA, M.J.A., Sept. 14, 2020)
}

\begin{abstract}
We summarize and announce some recent results initiating spectral analysis on pseudo-Riemannian locally symmetric spaces $\Gamma \backslash G / H$, beyond the classical setting where $H$ is compact (e.g. theory of automorphic forms for arithmetic $\Gamma$ ) or $\Gamma$ is trivial (e.g. Plancherel-type formula for semisimple symmetric spaces).
\end{abstract}

Key words: Locally symmetric space; pseudo-Riemannian manifold; discontinuous group; invariant differential operator; branching law; spherical variety.

1. Introduction. A pseudo-Riemannian manifold is a smooth manifold $M$ equipped with a smooth, nondegenerate symmetric bilinear tensor $g$ of signature $(p, q)$. It is called Riemannian if $q=0$, and Lorenzian if $q=1$. As in the Riemannian case, the metric $g$ induces a Radon measure on $M$ and a second-order differential operator

$$
\square_{M}=\operatorname{div} \operatorname{grad}
$$

called the Laplacian. It is a symmetric operator on the Hilbert space $L^{2}(M)$. The Laplacian $\square_{M}$ is not an elliptic differential operator if $p, q>0$.

A semisimple symmetric space $X$ is a homogeneous space $G / H$ where $G$ is a semisimple Lie group and $H$ an open subgroup of the group of fixed points of $G$ under some involutive automorphism. The manifold $X$ carries a $G$-invariant pseudoRiemannian metric induced by the Killing form of the Lie algebra $\mathfrak{g}$ of $G$. The group $G$ acts on $X$ by isometries, and the $\mathbf{C}$-algebra $\mathbf{D}_{G}(X)$ of $G$-invariant differential operators on $X$ is commutative.

In this note we consider quotients $X_{\Gamma}=\Gamma \backslash X$ of a semisimple symmetric space $X=G / H$ by discrete subgroups $\Gamma$ of $G$ acting properly discontinuously and freely on $X$ ("discontinuous groups for $X$ "). Such quotients are called pseudo-Riemannian locally symmetric spaces. They are complete

2020 Mathematics Subject Classification. Primary 22E40; Secondary 22E46, 58J50, 11F72, 53C 35 .

*) CNRS and Laboratoire Alexander Grothendieck, IHES, Université Paris-Saclay, 35 route de Chartres, 91440 Bures-surYvette, France.

**) Graduate School of Mathematical Sciences, The University of Tokyo, 3-8-1 Komaba, Tokyo 153-8914, Japan.

***) Kavli Institute for the Physics and Mathematics of the Universe (WPI), 5-1-5 Kashiwanoha, Kashiwa, Chiba 277-8583, Japan.
$(G, X)$-manifolds in the sense of Ehresmann and Thurston, and they inherit a pseudo-Riemannian structure from $X$. Any $G$-invariant differential operator $D$ on $X$ induces a differential operator $D_{\Gamma}$ on $X_{\Gamma}$ via the covering map $p_{\Gamma}: X \rightarrow X_{\Gamma}$. For instance, the Laplacian $\square_{X}$ on $X$ is $G$-invariant, and $\left(\square_{X}\right)_{\Gamma}=\square_{X_{\Gamma}}$. As in $[7,8]$, we think of

$$
\mathcal{P}:=\left\{D_{\Gamma}: D \in \mathbf{D}_{G}(X)\right\}
$$

as the set of "intrinsic differential operators" on the locally symmetric space $X_{\Gamma}$. It is a subalgebra of the $\mathbf{C}$-algebra $\mathbf{D}\left(X_{\Gamma}\right)$ of differential operators on $X_{\Gamma}$ :

$$
\mathbf{D}_{G}(X) \stackrel{\sim}{\rightarrow} \mathcal{P} \subset \mathbf{D}\left(X_{\Gamma}\right), \quad D \mapsto D_{\Gamma} .
$$

For a $\mathbf{C}$-algebra homomorphism $\lambda: \mathbf{D}_{G}(X) \rightarrow$ $\mathbf{C}$, we denote by $C^{\infty}\left(X_{\Gamma} ; \mathcal{M}_{\lambda}\right)$ the space of smooth functions $f$ on $X_{\Gamma}$ (joint eigenfunctions) satisfying the following system of partial differential equations:

$$
\left(\mathcal{M}_{\lambda}\right) \quad D_{\Gamma} f=\lambda(D) f \quad \text { for all } D \in \mathbf{D}_{G}(X) .
$$

Let $L^{2}\left(X_{\Gamma} ; \mathcal{M}_{\lambda}\right)$ be the space of square-integrable functions on $X_{\Gamma}$ satisfying $\left(\mathcal{M}_{\lambda}\right)$ in the weak sense. It is a closed subspace of the Hilbert space $L^{2}\left(X_{\Gamma}\right)$. We are interested in the following problems.

Problems 1. For intrinsic differential operators on $X_{\Gamma}=\Gamma \backslash G / H$,

(1) construct joint eigenfunctions on $X_{\Gamma}$;

(2) find a spectral theory on $L^{2}\left(X_{\Gamma}\right)$.

In the classical setting where $H$ is a maximal compact subgroup $K$ of $G$, i.e. $X_{\Gamma}$ is a Riemannian locally symmetric space, a rich and deep theory has been developed over several decades, in particular, in connection with automorphic forms when $\Gamma$ is arithmetic. For compact $H$, the spectral decompo- 
sition of $L^{2}\left(X_{\Gamma}\right)$ is closely related to a disintegration of the regular representation of $G$ on $L^{2}(\Gamma \backslash G)$ :

$$
L^{2}(\Gamma \backslash G) \simeq \int_{\widehat{G}}^{\oplus} m_{\Gamma}(\pi) \pi \mathrm{d} \sigma(\pi),
$$

where $\mathrm{d} \sigma$ is a Borel measure on the unitary dual $\widehat{G}$ and $m_{\Gamma}: \widehat{G} \rightarrow \mathbf{N} \cup\{\infty\}$ a measurable function called multiplicity. There is a natural isomorphism

$$
L^{2}\left(X_{\Gamma}\right) \simeq L^{2}(\Gamma \backslash G)^{H}
$$

and the Hilbert space $L^{2}\left(X_{\Gamma}\right)$ is decomposed as

$$
L^{2}\left(X_{\Gamma}\right) \simeq \int_{(\widehat{G})_{H}} m_{\Gamma}(\pi) \pi^{H} \mathrm{~d} \sigma(\pi),
$$

where $\pi^{H}$ denotes the space of $H$-invariant vectors in the representation space of $\pi$ and

$$
(\widehat{G})_{H}:=\left\{\pi \in \widehat{G}: \pi^{H} \neq\{0\}\right\} .
$$

Since the center $\mathfrak{Z}\left(\mathfrak{g}_{\mathbf{C}}\right)$ of the universal enveloping algebra $U\left(\mathfrak{g}_{\mathbf{C}}\right)$ acts on the space of smooth vectors of $\pi$ as scalars for every $\pi \in \widehat{G}$, the decomposition (1.4) respects the actions of $\mathbf{D}_{G}(X)$ and $\mathfrak{Z}\left(\mathfrak{g}_{\mathbf{C}}\right)$ via the natural C-algebra homomorphism $\mathrm{d} \ell: \mathfrak{Z}\left(\mathfrak{g}_{\mathbf{C}}\right) \rightarrow$ $\mathbf{D}_{G}(X)$. This homomorphism is surjective e.g. if $G$ is a classical group.

The situation changes drastically beyond the aforementioned classical setting, namely, when $H$ is not compact anymore. New difficulties include:

(1) (Representation theory) If $H$ is noncompact, then $L^{2}(\Gamma \backslash G)^{H}=\{0\}$ (because the fact that $\Gamma$ acts properly on $X=G / H$ implies that $H$ acts properly on $\Gamma \backslash G$ ), and so (1.3) fails:

$$
L^{2}\left(X_{\Gamma}\right) \not L^{2}(\Gamma \backslash G)^{H}
$$

and the irreducible decomposition (1.2) of the regular representation $L^{2}(\Gamma \backslash G)$ of $G$ does not yield a spectral decomposition of $L^{2}\left(X_{\Gamma}\right)$.

(2) (Analysis) In contrast to the usual Riemannian case (see [22]), the Laplacian $\square_{X_{\Gamma}}$ is not elliptic anymore, and thus even the following subproblems of Problem 1.(2) are open in general for $X_{\Gamma}=\Gamma \backslash G / H$ with $H$ noncompact.

Questions 2.

(1) Does the Laplacian $\square_{X_{\Gamma}}$, defined on $C_{c}^{\infty}\left(X_{\Gamma}\right)$, extend to a self-adjoint operator on $L^{2}\left(X_{\Gamma}\right)$ ?

(2) Does $L^{2}\left(X_{\Gamma} ; \mathcal{M}_{\lambda}\right)$ contain real analytic functions as a dense subspace?

(3) Does $L^{2}\left(X_{\Gamma}\right)$ decompose discretely into a sum of subspaces $L^{2}\left(X_{\Gamma} ; \mathcal{M}_{\lambda}\right)$ when $X_{\Gamma}$ is compact? Detailed proofs of Theorems 9, 10, 11, 15, and
16 below will appear elsewhere.

2. Standard quotients. We observe that a discrete group of isometries on a pseudoRiemannian manifold $X$ does not always act properly discontinuously on $X$, and the quotient space $X_{\Gamma}=\Gamma \backslash X$ is not necessarily Hausdorff. In fact, some semisimple symmetric spaces $X$ do not admit infinite discontinuous groups of isometries (Calabi-Markus phenomenon $[2,11]$ ), and thus it is not obvious a priori whether there are interesting examples of pseudo-Riemannian locally symmetric spaces $X_{\Gamma}$ beyond the classical Riemannian case.

Fortunately, there exist semisimple symmetric spaces $X=G / H$ admitting "large" discontinuous groups $\Gamma$, e.g. such that $X_{\Gamma}$ is compact or of finite volume. Let us recall a useful idea for finding such $X$ and $\Gamma$. Suppose a Lie subgroup $L$ of $G$ acts properly on $X$. Then the action of any discrete subgroup $\Gamma$ of $L$ on $X$ is automatically properly discontinuous, and this action is free whenever $\Gamma$ is torsion-free. Moreover, if $L$ acts cocompactly (e.g. transitively) on $X$, then $\operatorname{vol}\left(X_{\Gamma}\right)<+\infty$ if and only if $\operatorname{vol}(\Gamma \backslash L)<+\infty$.

Definition 3 (Standard quotient $X_{\Gamma}$, see [8, Def. 1.4]). A quotient $X_{\Gamma}=\Gamma \backslash X$ of $X=G / H$ by a discrete subgroup of $G$ is called standard if $\Gamma$ is contained in a reductive subgroup $L$ of $G$ acting properly on $X$.

A criterion on triples $(G, L, H)$ of reductive Lie groups for $L$ to act properly on $X=G / H$ was established in [11], and a list of irreducible symmetric spaces $G / H$ admitting proper and cocompact actions of reductive subgroups $L$ was given in [18]. Recently, Tojo [23] announced that the list in [18] exhausts all such triples $(L, G, H)$ with $L$ maximal.

3. Construction of discrete spectrum. Let $X=G / H$ be a semisimple symmetric space. Let $\mathfrak{j}$ be a maximal semisimple abelian subspace in the orthogonal complement of $\mathfrak{h}$ in $\mathfrak{g}$ with respect to the Killing form, and let $W$ be the Weyl group for the root system $\Sigma\left(\mathfrak{g}_{\mathbf{C}}, \mathfrak{j}_{\mathbf{C}}\right)$. The Harish-Chandra isomorphism $\Psi: S\left(\mathfrak{j}_{\mathbf{C}}\right)^{W} \stackrel{\sim}{\rightarrow} \mathbf{D}_{G}(X)$ (see [6]) induces a bijection

$$
\Psi^{*}: \operatorname{Hom}_{\mathbf{C}-a l g}\left(\mathbf{D}_{G}(X), \mathbf{C}\right) \stackrel{\sim}{\longrightarrow} \mathbf{j}_{\mathbf{C}}^{*} / W .
$$

The dimension of $\mathfrak{j}$ is called the rank of the symmetric space $X=G / H$. Let $K$ be a maximal compact subgroup of $G$ such that $H \cap K$ is a maximal compact subgroup of $H$. Assume that $G$ 
is connected without compact factor and that the following rank condition is satisfied:

$$
\operatorname{rank} G / H=\operatorname{rank} K /(H \cap K) .
$$

Then we can take $\mathfrak{j}$ to be a subspace of $\mathfrak{k}$. We fix compatible positive systems $\Sigma^{+}\left(\mathfrak{g}_{\mathbf{C}}, \mathfrak{j}_{\mathbf{C}}\right)$ and $\Sigma^{+}\left(\mathfrak{k}_{\mathbf{C}}, \mathfrak{j}_{\mathbf{C}}\right)$, denote by $\rho$ and $\rho_{c}$ the corresponding half sums of positive roots counted with multiplicities, and set

$$
\Lambda:=2 \rho_{c}-\rho+\mathbf{Z} \text {-span }\left\{\text { highest weights of }(\widehat{K})_{H \cap K}\right\} .
$$

For $C \geq 0$, we consider the countable set

$$
\Lambda_{C}:=\left\{\lambda \in \Lambda:\langle\lambda, \alpha\rangle>C \text { for all } \alpha \in \Sigma^{+}\left(\mathfrak{g}_{\mathbf{C}}, \mathfrak{j}_{\mathbf{C}}\right)\right\} .
$$

Fact 4 (Flensted-Jensen [5]). If the rank condition (3.2) holds, then there exists $C>0$ such that

$$
L^{2}\left(X ; \mathcal{M}_{\lambda}\right) \neq\{0\} \quad \text { for all } \lambda \in \Lambda_{C} .
$$

In fact one can take $C=0$ [19]. We now turn to locally symmetric spaces $X_{\Gamma}$ :

Theorem 5 ([7], [8, Th. 1.5]). Under the rank condition (3.2), for any standard quotient $X_{\Gamma}$ with $\Gamma$ torsion-free, there exists $C_{\Gamma}>0$ such that

$$
L^{2}\left(X_{\Gamma} ; \mathcal{M}_{\lambda}\right) \neq\{0\} \quad \text { for all } \lambda \in \Lambda_{C_{\Gamma}} .
$$

Thus the discrete spectrum $\operatorname{Spec}_{d}\left(X_{\Gamma}\right)$, which is by definition the set of $\lambda \in \operatorname{Hom}_{\mathbf{C}-a l g}\left(\mathbf{D}_{G}(X), \mathbf{C}\right)$ such that $L^{2}\left(X_{\Gamma} ; \mathcal{M}_{\lambda}\right) \neq\{0\}$, is infinite.

Theorem 5 applied to $(G \times\{1\}, G \times G, \operatorname{Diag} G)$ instead of $(L, G, H)$ (group manifold case) implies:

Example 6. Suppose $\operatorname{rank} G=\operatorname{rank} K$. For any torsion-free discrete subgroup $\Gamma$ and any discrete series representation $\pi_{\lambda}$ of $G$ with sufficiently regular Harish-Chandra parameter $\lambda$,

$$
\operatorname{Hom}_{G}\left(\pi_{\lambda}, L^{2}(\Gamma \backslash G)\right) \neq\{0\} .
$$

This sharpens and generalizes classical results asserting that if $\Gamma$ is an arithmetic subgroup of $G$, then (3.3) holds after replacing $\Gamma$ by a finite-index subgroup $\Gamma^{\prime}$ (possibly depending on $\pi_{\lambda}$ ), see BorelWallach [1], Clozel [3], DeGeorge-Wallach [4], Kazhdan [10], Rohlfs-Speh [20], and Savin [21].

Remark 7. (1) Theorem 5 extends to a more general setting where $X_{\Gamma}$ is not necessarily standard: namely, the conclusion still holds as long as the action of $\Gamma$ on $X$ satisfies a strong properness condition called sharpness [8, Th. 3.8].

(2) The rank condition (3.2) is necessary for $\operatorname{Spec}_{d}(X)$ to be nonempty (see Matsuki-Oshima
[19]), in which case Fact 4 applies. On the other hand, $\operatorname{Spec}_{d}\left(X_{\Gamma}\right)$ may be nonempty even if (3.2) fails. This leads us to the notion of discrete spectrum of type I and II, see Definition 12 below.

4. Spectral decomposition of $L^{2}\left(X_{\Gamma}\right)$. In this section, we discuss spectral decomposition on standard quotients $X_{\Gamma}$. We do not impose the rank condition (3.2), but require that $L_{\mathbf{C}}$ act spherically on $X_{\mathbf{C}}$, i.e. a Borel subgroup of $L_{\mathbf{C}}$ has an open orbit in $X_{\mathbf{C}}$. To be precise, our setting is as follows:

Setting 8. We consider a symmetric space $X=G / H$ with $G$ noncompact and simple, a reductive subgroup $L$ of $G$ acting properly on $X$ such that $X_{\mathbf{C}}=G_{\mathbf{C}} / H_{\mathbf{C}}$ is $L_{\mathbf{C}}$-spherical, and a torsion-free discrete subgroup $\Gamma$ of $L$.

For compact $H$, we can take $L=G$. However, our main interest is for noncompact $H$, in which case the proper action of $L$ in the setting 8 forces $L \neq G$ (see [11, Th. 4.1] for a properness criterion).

In Theorems 9 and 10 below, we allow the case where $\operatorname{vol}\left(X_{\Gamma}\right)=+\infty$.

Theorem 9 (Spectral decomposition). In the setting 8 , there exist a measure $\mathrm{d} \mu$ on Hom := $\operatorname{Hom}_{\mathbf{C}-a l g}\left(\mathbf{D}_{G}(X), \mathbf{C}\right)$ and a measurable family $\left(\mathcal{F}_{\lambda}\right)_{\lambda \in \text { Hom }}$ of linear maps, with

$$
\mathcal{F}_{\lambda}: C_{c}^{\infty}\left(X_{\Gamma}\right) \longrightarrow C^{\infty}\left(X_{\Gamma} ; \mathcal{M}_{\lambda}\right)
$$

such that any $f \in C_{c}^{\infty}\left(X_{\Gamma}\right)$ can be expanded into joint eigenfunctions on $X_{\Gamma}$ as

$$
f=\int_{\text {Hom }} \mathcal{F}_{\lambda} f \mathrm{~d} \mu(\lambda),
$$

with a Parseval-Plancherel type formula

$$
\|f\|_{L^{2}\left(X_{\Gamma}\right)}^{2}=\int_{\text {Hom }}\left\|\mathcal{F}_{\lambda} f\right\|_{L^{2}\left(X_{\Gamma}\right)}^{2} \mathrm{~d} \mu(\lambda) .
$$

The measure $\mathrm{d} \mu$ can be described via a "transfer map" discussed in Section 5, see (5.4). In particular, we see that (4.1) is a discrete sum if $X_{\Gamma}$ is compact, answering Question 2.(3) in our setting. The proof of Theorem 9 gives an answer to Questions 2.(1)-(2):

Theorem 10. In the setting 8 ,

(1) the pseudo-Riemannian Laplacian $\square_{X_{\Gamma}}$ defined on $C_{c}^{\infty}\left(X_{\Gamma}\right)$ is essentially self-adjoint on $L^{2}\left(X_{\Gamma}\right)$;

(2) any $L^{2}$-eigenfunction of the Laplacian $\square_{X_{\Gamma}}$ can be approximated by real analytic $L^{2}$-eigenfunctions.

Theorem 11. In the setting 8 , the discrete spectrum $\operatorname{Spec}_{d}\left(X_{\Gamma}\right)$ is infinite whenever $\Gamma$ is cocompact or arithmetic in the subgroup $L$. 
Let $\mathcal{D}^{\prime}(X)$ be the space of distributions on $X$, endowed with its standard topology. Let $p_{\Gamma}^{*}: L^{2}\left(X_{\Gamma}\right) \rightarrow \mathcal{D}^{\prime}(X)$ be the pull-back by the projection $p_{\Gamma}: X \rightarrow X_{\Gamma}$. For $\lambda \in \operatorname{Spec}_{d}\left(X_{\Gamma}\right)$, we denote by $L^{2}\left(X_{\Gamma} ; \mathcal{M}_{\lambda}\right)_{\mathbf{I}}$ the preimage under $p_{\Gamma}^{*}$ of the closure in $\mathcal{D}^{\prime}(X)$ of $L^{2}\left(X_{\Gamma} ; \mathcal{M}_{\lambda}\right)$, and by $L^{2}\left(X_{\Gamma} ; \mathcal{M}_{\lambda}\right)_{\mathrm{II}}$ its orthogonal complement in $L^{2}\left(X_{\Gamma} ; \mathcal{M}_{\lambda}\right)$.

Definition 12. For $i=\mathbf{I}$ or II, the discrete spectrum of type $i$ of $X_{\Gamma}$ is the subset $\operatorname{Spec}_{d}\left(X_{\Gamma}\right)_{i}$ of $\operatorname{Spec}_{d}\left(X_{\Gamma}\right)$ consisting of those elements $\lambda$ such that $L^{2}\left(X_{\Gamma} ; \mathcal{M}_{\lambda}\right)_{i} \neq\{0\}$

By construction, $\operatorname{Spec}_{d}\left(X_{\Gamma}\right)_{\mathbf{I}}$ is contained in $\operatorname{Spec}_{d}(X)$, hence it is nonempty only if (3.2) holds (Remark 7.(2)); in this case $\operatorname{Spec}_{d}\left(X_{\Gamma}\right)_{\mathbf{I}}$ is actually infinite for standard $X_{\Gamma}$ by Theorem 5. On the other hand, Theorem 11 has the following refinement.

Theorem 13. In the setting $8, \operatorname{Spec}_{d}\left(X_{\Gamma}\right)_{\mathrm{II}}$ is infinite whenever $\Gamma$ is cocompact or arithmetic in $L$.

Example 14. For any compact standard anti-de Sitter 3-manifold $M=\Gamma \backslash \mathrm{SO}(2,2) / \mathrm{SO}(2,1)$, both $\operatorname{Spec}_{d}\left(X_{\Gamma}\right)_{\mathrm{I}}$ and $\operatorname{Spec}_{d}\left(X_{\Gamma}\right)_{\mathrm{II}}$ are infinite, and

$$
\operatorname{Spec}_{d}\left(X_{\Gamma}\right)_{\mathbf{I}} \subset[0,+\infty), \quad \operatorname{Spec}_{d}\left(X_{\Gamma}\right)_{\mathbf{I I}} \subset(-\infty, 0] .
$$

5. Transfer maps. Let $L$ be a reductive subgroup of $G$ acting properly on $X=G / H$ and $\Gamma$ a discrete subgroup of $L$. In Section 1 we considered spectral analysis on the standard locally symmetric space $X_{\Gamma}$ through the algebra $\mathcal{P}\left(\simeq \mathbf{D}_{G}(X)\right)$ of intrinsic differential operators on $X_{\Gamma}$. Another C-algebra $\mathcal{Q}$ of differential operators on $X_{\Gamma}$ is obtained from the center $\mathfrak{Z}\left(\mathfrak{l}_{\mathbf{C}}\right)$ of the enveloping algebra $U\left(\mathfrak{l}_{\mathbf{C}}\right)$ : indeed, $\mathfrak{Z}\left(\mathfrak{l}_{\mathbf{C}}\right)$ acts on smooth functions on $X$ by differentiation, yielding a $\mathbf{C}$-algebra of $L$-invariant differential operators on $X$, hence a C-algebra of differential operators on $X_{\Gamma}=\Gamma \backslash X$ since $\Gamma \subset L$. In general, there is no inclusion relation between $\mathcal{P}$ and $\mathcal{Q}$. In order to compare the roles of $\mathcal{P}$ and $\mathcal{Q}$, we highlight a natural homomorphism $\mathfrak{Z}\left(\mathfrak{g}_{\mathrm{C}}\right) \rightarrow \mathcal{P}$ and a surjective one $\mathrm{d} \ell: \mathfrak{Z}\left(\mathfrak{l}_{\mathbf{C}}\right) \rightarrow \mathcal{Q}$. Loosely speaking, the algebras $\mathfrak{Z}\left(\mathfrak{g}_{\mathbf{C}}\right)$ and $\mathfrak{Z}\left(\mathfrak{l}_{\mathbf{C}}\right)$ separate irreducible representations of the groups $G$ and $L$, respectively, hence it is important to understand how irreducible representations of $G$ behave when restricted to the subgroup $L$ (branching problem) in order to utilize the algebra $\mathcal{Q}$ for the spectral analysis on $X_{\Gamma}$ via the algebra $\mathcal{P}$ (see $[15,16]$ ). We shall return to this point in Theorem 15 below.
Now assume the proper action of $L$ on $X=$ $G / H$ is also transitive, so that $X \simeq L / L_{H}$ where $L_{H}:=L \cap H$ is compact. Up to conjugation, we may assume that $L_{K}:=L \cap K$ is a maximal compact subgroup of $L$ containing $L_{H}$. Then the pseudo-Riemannian symmetric space $X$ fibers over the Riemannian symmetric space $Y=L / L_{K}$ with fiber $F:=L_{K} / L_{H}$, and this induces a fibration for the quotients by $\Gamma$ :

$$
F \longrightarrow X_{\Gamma} \simeq \Gamma \backslash L / L_{H} \longrightarrow Y_{\Gamma}=\Gamma \backslash L / L_{K} .
$$

To expand functions on $X_{\Gamma}$ along the fiber $F$, we define an endomorphism $p_{\tau}$ of $C^{\infty}\left(X_{\Gamma}\right)$ by

$$
\left(p_{\tau} f\right)(\cdot):=\frac{1}{\operatorname{dim} \tau} \int_{K} f(\cdot k) \operatorname{Trace} \tau(k) \mathrm{d} k
$$

for every $\tau \in \widehat{L_{K}}$. Then $p_{\tau}$ is an idempotent, namely, $p_{\tau}^{2}=p_{\tau}$. The $\tau$-component of $C^{\infty}\left(X_{\Gamma}\right)$ is defined by

$$
C^{\infty}\left(X_{\Gamma}\right)_{\tau}:=\operatorname{Image}\left(p_{\tau}\right)=\operatorname{Ker}\left(p_{\tau}-\mathrm{id}\right) .
$$

We note that $C^{\infty}\left(X_{\Gamma}\right)_{\tau} \neq\{0\}$ if and only if $\tau$ has a nonzero $L_{H}$-invariant vector, i.e. $\tau \in\left(\widehat{\bar{L}_{K}}\right)_{L_{H}}$. It is easy to see that the projection $p_{\tau}$ commutes with any element in $\mathcal{Q}\left(\simeq \mathrm{d} \ell\left(\mathcal{Z}\left(\mathfrak{l}_{\mathbf{C}}\right)\right)\right)$, but not always with "intrinsic differential operators" $D_{\Gamma} \in$ $\mathcal{P}\left(\simeq \mathbf{D}_{G}(X)\right)$, and consequently it may well happen that

$$
p_{\tau}\left(C^{\infty}\left(X_{\Gamma} ; \mathcal{M}_{\lambda}\right)\right) \not \subset C^{\infty}\left(X_{\Gamma} ; \mathcal{M}_{\lambda}\right) .
$$

To make a connection between the two subalgebras $\mathcal{P}$ and $\mathcal{Q}$, we introduce a third subalgebra $\mathcal{R}$ of $\mathbf{D}\left(X_{\Gamma}\right)$, coming from the fiber $F$ in (5.1). Namely, $\mathcal{R}$ is isomorphic to the $\mathbf{C}$-algebra $\mathbf{D}_{L_{K}}(F)$ of $L_{K}$-invariant differential operators $D$ on $F$, and obtained by extending elements of $\mathbf{D}_{L_{K}}(F)$ to $L$-invariant differential operators on $X$, yielding differential operators on the quotient $X_{\Gamma}$.

Suppose now that we are in the setting 8 . The subgroup $L$ acts transitively on $X$ by [17, Lem. 4.2] and [12, Lem. 5.1]. Moreover, we can prove [9] that

$$
\mathcal{Q} \subset\langle\mathcal{P}, \mathcal{R}\rangle
$$

where $\langle\mathcal{P}, \mathcal{R}\rangle$ denotes the subalgebra of $\mathbf{D}\left(X_{\Gamma}\right)$ generated by $\mathcal{P}$ and $\mathcal{R}$. This implies the following strong constraints on the restriction of representations:

Theorem 15. In the setting 8 , any irreducible $(\mathfrak{g}, K)$-module occurring in $C^{\infty}(X)$ is discretely decomposable as an $(\mathfrak{l}, L \cap K)$-module. 
See [12-14] for a general theory of discretely decomposable restrictions of representations. See also [16] for a discussion on Theorem 15 when dropping the assumption that $L$ acts properly on $X$.

In addition to (5.2), the quotient fields of $\mathcal{P}$ and $\langle\mathcal{Q}, \mathcal{R}\rangle$ coincide $[9$, Th. $1.3 \& \S 6.9]$, and we obtain:

Theorem 16 (Transfer map). In the setting 8 , for any $\tau \in\left(\widehat{L_{K}}\right)_{L_{H}}$ there is an injective map

$$
\boldsymbol{\nu}(\cdot, \tau): \operatorname{Hom}_{\mathbf{C}-\text { alg }}\left(\mathbf{D}_{G}(X), \mathbf{C}\right) \hookrightarrow \operatorname{Hom}_{\mathbf{C}-\text { alg }}\left(\mathfrak{Z}\left(\mathfrak{l}_{\mathbf{C}}\right), \mathbf{C}\right)
$$

such that for any $\lambda \in \operatorname{Hom}_{\mathbf{C} \text {-alg }}\left(\mathbf{D}_{G}(X), \mathbf{C}\right)$, any $f \in$ $C^{\infty}\left(X_{\Gamma} ; \mathcal{M}_{\lambda}\right)$, and any $z \in \mathfrak{Z}\left(\mathfrak{l}_{\mathbf{C}}\right)$,

$$
\mathrm{d} \ell(z)\left(p_{\tau} f\right)=\boldsymbol{\nu}(\lambda, \tau)(z) p_{\tau} f .
$$

We write $\boldsymbol{\lambda}(\cdot, \tau)$ for the inverse map of $\boldsymbol{\nu}(\cdot, \tau)$ on its image. We call $\boldsymbol{\nu}(\cdot, \tau)$ and $\boldsymbol{\lambda}(\cdot, \tau)$ transfer maps, as they "transfer" eigenfunctions for $\mathcal{P}$ to those for $\mathcal{Q}$, and vice versa, on the $\tau$-component $C^{\infty}\left(X_{\Gamma}\right)_{\tau}$. let

For an explicit description of transfer maps,

$$
\Phi^{*}: \operatorname{Hom}_{\mathbf{C}-a l g}\left(\mathfrak{Z}\left(\mathfrak{l}_{\mathbf{C}}\right), \mathbf{C}\right) \stackrel{\sim}{\rightarrow} \mathfrak{t}_{\mathbf{C}}^{*} / W\left(\mathfrak{l}_{\mathbf{C}}\right)
$$

be the Harish-Chandra isomorphism as in (3.1), where $W\left(\mathfrak{l}_{\mathbf{C}}\right)$ denotes the Weyl group of the root system $\Delta\left(\mathfrak{l}_{\mathbf{C}}, \mathfrak{t}_{\mathbf{C}}\right)$ with respect to a Cartan subalgebra $\mathfrak{t}_{\mathbf{C}}$ in $\mathfrak{l}_{\mathbf{C}}$. We note that there is no natural inclusion relation between $\mathfrak{j}_{\mathbf{C}}$ and $\mathfrak{t}_{\mathbf{C}}$.

For each $\tau \in\left(\widehat{L_{K}}\right)_{L_{H}}$, we find an affine map $S_{\tau}: \mathbf{j}_{\mathbf{C}}^{*} \rightarrow \mathfrak{t}_{\mathbf{C}}^{*}$ such that the following diagram commutes:

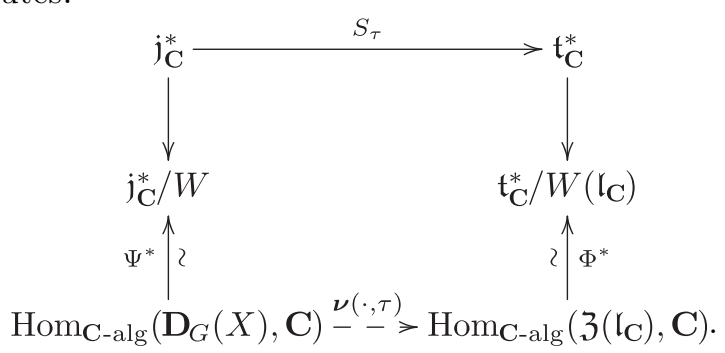

A closed formula for the transfer map $\boldsymbol{\nu}(\cdot, \tau)$ is derived from that of the affine map $S_{\tau}$, which was determined explicitly in $[9, \S 6-7]$ for the complexifications of the triples $(L, G, H)$ in the setting 8 .

Via the transfer maps, we can utilize representations of the subgroup $L$ efficiently for the spectral analysis on $X_{\Gamma}$, as follows. As in (1.2), let

$$
L^{2}(\Gamma \backslash L) \simeq \int_{\widehat{L}}^{\oplus} m_{\Gamma}(\vartheta) \vartheta \mathrm{d} \sigma(\vartheta)
$$

be a disintegration of the regular representation $L^{2}(\Gamma \backslash L)$ of the subgroup $L$. Then the transform $\mathcal{F}_{\lambda}$ in Theorem 9 can be built naturally by using (5.3) and the expansion of $C_{c}^{\infty}\left(X_{\Gamma}\right)$ along the fiber $F$ in (5.1). Consider the map

$$
\Lambda:(\widehat{L})_{L_{H}} \times\left(\widehat{L_{K}}\right)_{L_{H}} \rightarrow \operatorname{Hom}_{\mathbf{C}-a l g}\left(\mathbf{D}_{G}(X), \mathbf{C}\right),
$$

$(\vartheta, \tau) \mapsto \boldsymbol{\lambda}\left(\chi_{\vartheta}, \tau\right)$, where $\chi_{\vartheta} \in \operatorname{Hom}_{\mathbf{C} \text {-alg }}\left(\mathfrak{Z}\left(\mathfrak{l}_{\mathbf{C}}\right), \mathbf{C}\right)$ is the infinitesimal character of $\vartheta \in \widehat{L}$. Then the Plancherel measure $\mathrm{d} \mu$ on $\operatorname{Hom}_{\mathbf{C} \text {-alg }}\left(\mathbf{D}_{G}(X), \mathbf{C}\right)$ in Theorem 9 can be defined by

$$
\mathrm{d} \mu=\Lambda_{*}\left(\left.\mathrm{~d} \sigma\right|_{(\widehat{L})_{L_{H}}} \times\left(\widehat{L_{K}}\right)_{L_{H}}\right) .
$$

Acknowledgements. This project received funding from the European Research Council under the European Union's Horizon 2020 research and innovation programme (ERC starting grant DiGGeS, grant agreement No. 715982), and from JSPS Kakenhi Grant Number JP18H03669. We also acknowledge the hospitality of IHES.

We thank the referee for helpful comments.

\section{References}

[ 1 ] A. Borel and N. Wallach, Continuous cohomology, discrete subgroups, and representations of reductive groups, 2nd. ed., Mathematical Surveys and Monographs, 67, American Mathematical Society, Providence, RI, 2000.

[ 2 ] E. Calabi and L. Markus, Relativistic space forms, Ann. of Math. 75 (1962), 63-76.

[ 3 ] L. Clozel, On limit multiplicities of discrete series representations in spaces of automorphic forms, Invent. Math. 83 (1986), no. 2, 265-284.

[ 4 ] D. L. de George and N. R. Wallach, Limit formulas for multiplicities in $L^{2}(\Gamma \backslash G)$, Ann. of Math. (2) 107 (1978), no. 1, 133-150.

[ 5 ] M. Flensted-Jensen, Discrete series for semisimple symmetric spaces, Ann. of Math. 111 (1980), no. 2, 253-311.

[ 6 ] S. Helgason, Groups and geometric analysis, corrected reprint of the 1984 original, Mathematical Surveys and Monographs, 83, American Mathematical Society, Providence, RI, 2000.

[ 7 ] F. Kassel and T. Kobayashi, Stable spectrum for pseudo-Riemannian locally symmetric spaces, C. R. Math. Acad. Sci. Paris 349 (2011), no. 1$2,29-33$.

[ 8 ] F. Kassel and T. Kobayashi, Poincaré series for non-Riemannian locally symmetric spaces, Adv. Math. 287 (2016), 123-236.

[ 9 ] F. Kassel and T. Kobayashi, Invariant differential operators on spherical homogeneous spaces with overgroups, J. Lie Theory 29 (2019), no. 3, 663-754.

[10] D. Kazhdan, Some applications of the Weil representation, J. Analyse Mat. 32 (1977), 235-248.

[11 ] T. Kobayashi, Proper action on a homogeneous space of reductive type, Math. Ann. 285 (1989), 
no. 2, 249-263.

[12] T. Kobayashi, Discrete decomposability of the restriction of $A_{q}(\lambda)$ with respect to reductive subgroups and its applications, Invent. Math. 117 (1994), no. 2, 181-205.

[13] T. Kobayashi, Discrete decomposability of the restriction of $A_{q}(\lambda)$ with respect to reductive subgroups. II. Micro-local analysis and asymptotic $K$-support, Ann. of Math. (2) 147 (1998), no. 3, 709-729.

[14] T. Kobayashi, Discrete decomposability of the restriction of $A_{q}(\lambda)$ with respect to reductive subgroups. III. Restriction of Harish-Chandra modules and associated varieties, Invent. Math. 131 (1998), no. 2, 229-256.

[15] T. Kobayashi, Hidden symmetries and spectrum of the Laplacian on an indefinite Riemannian manifold, in Spectral analysis in geometry and number theory, Contemp. Math., 484, Amer. Math. Soc., Providence, RI, 2009, pp. 73-87,

[16 ] T. Kobayashi, Global analysis by hidden symmetry, in Representation theory, number theory, and invariant theory, Progr. Math., 323, Birkhäuser/Springer, Cham., 2017, pp. 359397.

[ 17 ] T. Kobayashi and T. Oshima, Finite multiplicity theorems for induction and restriction, Adv. Math. 248 (2013), 921-944.

[ 18 ] T. Kobayashi and T. Yoshino, Compact CliffordKlein forms of symmetric spaces-revisited, Pure Appl. Math. Q. 1 (2005), no. 3, Special Issue: In memory of Armand Borel. Part 2, 591663.

[19] T. Ōshima and T. Matsuki, A description of discrete series for semisimple symmetric spaces, in Group representations and systems of differential equations (Tokyo, 1982), 331-390, Adv. Stud. Pure Math., 4, North-Holland, Amsterdam, 1984

[ 20 ] J. Rohlfs and B. Speh, On limit multiplicities of representations with cohomology in the cuspidal spectrum, Duke Math. J. 55 (1987), no. 1, 199-211.

[21] G. Savin, Limit multiplicities of cusp forms, Invent. Math. 95 (1989), no. 1, 149-159.

[ 22 ] R. S. Strichartz, Analysis of the Laplacian on the complete Riemannian manifold, J. Functional Analysis 52 (1983), no. 1, 48-79.

[23] K. Tojo, Classification of irreducible symmetric spaces which admit standard compact CliffordKlein forms, Proc. Japan Acad. Ser. A Math. Sci. 95 (2019), no. 2, 11-15. 